\title{
Renormalization of a Quadratic Interaction in the Hamiltonian Formalism
}

\author{
J. GiNIBRE \\ Laboratoire de Physique Théorique, Faculté des Sciences d'Orsay, France \\ and \\ G. VELO \\ Istituto di Fisica dell'Università di Bologna
}

Received February 18, 1970

\begin{abstract}
The method of the dressing transformation is used to perform a mass renormalization of a neutral scalar free field in the Hamiltonian formalism, for arbitrary space dimension. The resulting situation is analyzed by means of a Bogoliubov transformation, and seen to yield the expected results.
\end{abstract}

\section{Introduction}

It is well known that quantum field theory is plagued with divergences which make the construction of mathematically meaningful models a formidable task. These divergences are of two types: ultraviolet (UV) divergences, connected with high momentum behaviour, and divergences connected with the infinite volume (Haag's theorem).

A significant progress in circumventing the first class of divergences has been made recently by Glimm $[1,2]$ using the hamiltonian formalism. He considered, among others, the case of a neutral scalar field $\Phi$ with a $\Phi^{4}$ interaction in three dimensional space time [2], with a space cutoff which eliminates the infinite volume divergences. The remaining UV divergences still make it impossible to define the Hamiltonian of the system in the original Fock space. However, Glimm was able to define a new Hilbert space in which a suitably renormalized version of the Hamiltonian makes sense as a symmetric operator. Unfortunately, the construction of the new Hilbert space and of the renormalized Hamiltonian is fairly complicated; in particular, it is not known whether the latter is semi-bounded and can be extended to a self-adjoint operator. Moreover, in higher dimensional space time or with more singular interactions, higher divergences occur, and it is not clear how to extend the method to such cases. It is therefore of interest to test the method on a simple model, namely the quadratic Hamiltonian [3,4], for which nevertheless arbitrarily high divergences occur if one takes the dimension

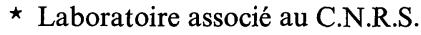


$s$ of the space sufficiently large. In particular, for $s \geqq 4$, UV divergences require a change of Hilbert space as in the $\Phi^{4}$ theory for $s=2$.

In this paper, we perform the renormalization of the quadratic Hamiltonian for arbitrary space dimension in a rigorous way. Starting from an interaction Hamiltonian with a space cut-off and a UV cut-off, we first apply Glimm's method to remove the UV cut-off and to define a new Hilbert space and renormalized Hamiltonian as a symmetric operator in this space (Section 2). In order to identify the new space and Hamiltonian we follow a different path and perform a Bogoliubov transformation, which almost diagonalizes the original Hamiltonian. This makes it possible to define another renormalized Hamiltonian in the Fock space of the Bogoliubov transformed of the original creation and annihilation operators. The two procedures turn out to be equivalent: in fact, there exists a unitary mapping from one Hilbert space to the other, such that the matrix elements of the operator defined by the first method correspond under this mapping to the matrix elements of the operator defined by the second method. This identification shows that the renormalized hamiltonian is positive and has a unique ground state (Section 3). We can then proceed to remove the space cut-off. It is remarkable that, for this limit, no further change of Hilbert space is required. The field operators and the Hamiltonian converge in the strong operator topology on a suitable domain, and we end up with a free field corresponding to the renormalized mass (Section 4). Technical verifications are collected in two appendices.

\section{Renormalization Using a Dressing Transformation}

In this section, we perform the renormalization of the quadratic Hamiltonian for a neutral scalar field of mass $m$ in $s$-dimensional space, following closely Glimm [2]. We use the following notations [5]: let $\mathfrak{H}$ be the Fock space; a vector $\psi$ in $\mathfrak{H}$ is a sequence $\left\{\psi(K) ; K=k_{1}, \ldots, k_{n}\right.$; $n=0,1,2, \ldots\}$ of symmetric square integrable functions of $n$ vectors in $R^{s}$. The scalar product is defined by:

$$
(\varphi, \psi)=f d K \bar{\varphi}(K) \psi(K)
$$

where

$$
f d K=\sum_{n=0}^{\infty} \frac{1}{n !} \int d k_{1} \ldots d k_{n} .
$$

The vacuum state is denoted by $\Omega$. We shall make use later on of the set $\mathscr{D} \subset \mathfrak{H}$ of vectors with a finite number of particles and with wave functions belonging to the Schwartz space $\mathscr{S}$. The creation and annihila- 
tion operators, the field operator and the free Hamiltonian are defined respectively by:

$$
\begin{gathered}
\left(a^{+}(k) \psi\right)(K)=\sum_{i=1}^{n} \delta\left(k-k_{i}\right) \psi\left(K_{i}\right), \\
(a(k) \psi)(K)=\psi(K, k)
\end{gathered}
$$

where $K_{i}$ is obtained from $K$ by omitting $k_{i}$.

$$
\begin{gathered}
\Phi(x)=(2 \pi)^{-s / 2} \int d k(2 \omega(k))^{-1 / 2} e^{i k x}\left(a(k)+a^{+}(-k)\right), \\
H_{0}=\int d k \omega(k) a^{+}(k) a(k)
\end{gathered}
$$

where $\omega(k)=\left(k^{2}+m^{2}\right)^{1 / 2}$.

The interaction Hamiltonian is given formally by:

$$
V=2 \lambda \int d x: \Phi(x)^{2}:
$$

and corresponds to a mass renormalization $\delta m^{2}=4 \lambda$.

For this operator to make sense in $\mathfrak{H}$, it is necessary to introduce a space cut-off and (for $s \geqq 2$ ), an ultra violet (UV) cut-off. Let $\hat{f}$ be a real positive even function in $\mathscr{S}$, normalized to $\hat{f}(0)=1$, with positive (even) Fourier transform $f$ defined by:

$$
f(k)=(2 \pi)^{-s} \int d x \hat{f}(x) e^{i k x}
$$

$f$ will serve as a space cut-off and will be kept fixed until Section 4, where we shall take the limit $\hat{f} \rightarrow 1$, i.e. $f \rightarrow \delta$. Let $\chi_{\sigma}(k)$ be a positive $\mathscr{C}^{\infty}$ function with compact support, equal to one for $|k|<\sigma$. The elimination of the UV cut-off consists in taking the limit $\sigma \rightarrow \infty$. Define:

$$
\begin{gathered}
\Phi_{\sigma}(x)=(2 \pi)^{-s / 2} \int d k(2 \omega(k))^{-1 / 2} \chi_{\sigma}(k) e^{i k x}\left(a(k)+a^{+}(-k)\right) \\
V_{\sigma}=2 \lambda \int d x \hat{f}(x): \Phi_{\sigma}(x)^{2}:
\end{gathered}
$$

$V_{\sigma}$ exists as an operator in $\mathfrak{H}$ and $H_{\sigma}=H_{0}+V_{\sigma}$ is essentially self adjoint on the domain of $H_{0}$ (Appendix 1). $V_{\sigma}$ can be decomposed as a sum:

where

$$
V_{\sigma}=V_{0 \sigma}+V_{1 \sigma}+V_{2 \sigma}
$$

$$
\begin{aligned}
& V_{0 \sigma}=V_{2 \sigma}^{+}=\lambda \int d k d l[\omega(k) \omega(l)]^{-1 / 2} f(k+l) \chi_{\sigma}(k) \chi_{\sigma}(l) a(k) a(l), \\
& V_{1 \sigma}=2 \lambda \int d k d l[\omega(k) \omega(l)]^{-1 / 2} f(k-l) \chi_{\sigma}(k) \chi_{\sigma}(l) a^{+}(k) a(l) .
\end{aligned}
$$

The UV divergences depend in an essential way on the dimension of the space. We expect the following situation to happen [6]: for $s=1$, no divergence occurs, $V_{\infty}$ exists as an operator on the domain of $H_{0}$, 
and no UV cut-off is needed. For $s=2, H_{\infty}$ is defined in $\mathfrak{H}$, but $\mathscr{D}\left(H_{\infty}\right)$ $\cap \mathscr{D}\left(H_{0}\right)=\{0\}$. For $s=3$, a change of domain is again necessary, and an infinite constant has to be added to the Hamiltonian. For $s \geqq 4$, it is no longer possible to define $H_{\infty}$ in $\mathfrak{H}$, and a change of Hilbert space is required.

In order to perform the change of domain or the change of Hilbert space, we introduce a dressing transformation $[2,5]$ :

$$
T_{\sigma}=\exp \left(-W_{\sigma}\right)
$$

where

$$
W_{\sigma}=\frac{1}{2} \int d k d l \tilde{w}_{\sigma}(k,-l) a^{+}(k) a^{+}(l)
$$

Here, $\tilde{w}_{\sigma}(k, l)$ is a real $L^{2}-$ kernel satisfying $\tilde{w}_{\sigma}(k, l)=\tilde{w}_{\sigma}(l, k)=\tilde{w}_{\sigma}(-k,-l)$, to be chosen below. $\tilde{w}_{\sigma}$ defines a bounded self adjoint operator in the one particle space $\mathfrak{H}_{1} \equiv L^{2}\left(R^{s}, d k\right)$, which we shall again denote by $\tilde{w}_{\sigma}$.

With this definition, the following identity holds:

$$
\left(H_{\sigma}+C_{\sigma}\right) T_{\sigma}=T_{\sigma}\left[H_{\sigma}-\left(H_{0}+V_{0 \sigma}+V_{1 \sigma}\right)+W_{\sigma}+\frac{1}{2} V_{0 \sigma} \frac{1}{2} W_{\sigma}^{2}\right]
$$

where $\frac{1}{p}$ denotes the connected product with $p$ contractions $[2,5]$ and:

$$
C_{\sigma}=V_{0 \sigma} \frac{1}{2} W_{\sigma} .
$$

Since we want to use (2.2) to define the renormalized Hamiltonian in the limit $\sigma \rightarrow \infty$, we choose $W_{\sigma}$ so as to eliminate the most singular terms in the RHS, namely, in the square bracket, the terms containing two creation operators. This gives the equation:

$$
R_{\sigma} \equiv V_{2 \sigma}-H_{0} \leftarrow W_{\sigma}-V_{1 \sigma} \leftarrow W_{\sigma}+\frac{1}{2} V_{0 \sigma} \frac{1}{2} W_{\sigma}^{2}=0 .
$$

In the next section and in Appendix 2, we shall prove that for $\lambda>-m^{2} / 4$, Eq. (2.4) has a solution $\tilde{w}_{\sigma}$ with the following properties: for $\sigma \leqq \infty$, $\tilde{w}_{\sigma}(k, l)$ is real symmetric bounded $\mathscr{C}^{\infty}$ function of $(k, l)$, and $\operatorname{Sup}_{\sigma}\left\|\tilde{w}_{\sigma}\right\|<1$. For $\sigma$ finite, $\tilde{w}_{\sigma}(k, l)$ has compact support in $(k, l)$, and therefore $\tilde{w}_{\sigma}$ is a Hilbert Schmidt operator: $\operatorname{Tr} \tilde{w}_{\sigma}^{2}<\infty$. For $\sigma$ infinite, $\operatorname{Tr} \tilde{w}^{2}$ can be shown to be infinite for $s \geqq 4$. When $\sigma$ tends to infinity, $\tilde{w}_{\sigma}$ tends to $\tilde{w}$ in the strong operator topology.

From now on, we choose this specific $\tilde{w}_{\sigma}$.

We shall now use $T_{\sigma}$ to define a new scalar product on a dense subset of $\mathfrak{H}$. For this purpose, it will be convenient to have an explicit expression for $\left(T_{\sigma} \psi, T_{\sigma} \varphi\right)$. To perform this calculation, the following notations are useful. Let $K=\left(k_{1}, \ldots, k_{n}\right)$ and $L=\left(l_{1}, \ldots, l_{m}\right)$. Let $\alpha \in L^{2}\left(R^{s}, d k\right)$ be a one particle wave function. We define a vector $E \alpha \in \mathfrak{H}$ by:

$$
E \alpha(K)=\prod_{i=1}^{n} \alpha\left(k_{i}\right)
$$


Let $\tilde{s}(k, l)$ be a symmetric function of two vectors $(k, l)$. We define (cf. [7]):

$$
\begin{aligned}
& E(\tilde{s})(K \mid L)=0 \quad \text { for } m \neq n \\
& =\sum_{n} \prod_{i=1}^{n} \tilde{s}\left(k_{i}, l_{\pi(i)}\right) \text { for } m=n
\end{aligned}
$$

where the sum runs over all permutations $\pi$ of $(1,2, \ldots, n)$.

We finally denote by $E(\tilde{S})(K)$ the sum over the partitions of $K$ in pairs of variables (this requires that $n$ be even for $E(\tilde{S})(K)$ to be non zero) of the products of the $\tilde{s}\left(k_{i},-k_{j}\right)$ for all the pairs of the partition.

We shall also use the notations $E(\tilde{s})(K \mid L)$ and $E(\tilde{s})(K)$ with $\tilde{s}$ an operator in $L^{2}\left(R^{s}, d k\right)$ represented by a symmetric even kernel $\tilde{s}(k, l)$.

We can now state the following result:

Lemma. Let $\sigma$ be finite, $\varphi \in \mathscr{D}, \psi \in \mathscr{D}$. Then:

$$
\begin{aligned}
&\left\|T_{\sigma} \Omega\right\|^{2}=\exp \left[-\frac{1}{2} \operatorname{Tr} \log \left(1-\tilde{w}_{\sigma}^{2}\right)\right] \\
& \frac{\left(T_{\sigma} \varphi, T_{\sigma} \psi\right)}{\left\|T_{\sigma} \Omega\right\|^{2}}= f d K d K^{\prime} d L d L^{\prime} \psi\left(K, K^{\prime}\right) \overline{\varphi\left(L, L^{\prime}\right)} \\
& \times E\left(-\tilde{w}_{\sigma}\left(1-\tilde{w}_{\sigma}^{2}\right)^{-1}\right)\left(K^{\prime}\right) E\left(-\tilde{w}_{\sigma}\left(1-\tilde{w}_{\sigma}^{2}\right)^{-1}\right)\left(L^{\prime}\right) \\
& \times E\left(\left(1-\tilde{w}_{\sigma}^{2}\right)^{-1}\right)(K \mid L) .
\end{aligned}
$$

Proof. In order to deal with the combinational problem, it is sufficient to consider the case where $\tilde{\mathrm{w}}_{\sigma}$ is a finite matrix and where a finite number of modes occur. For each mode, we introduce coherent states [8] $\left.\mid \xi_{k}\right)$ defined by $\left.\left.a_{k} \mid \xi_{k}\right)=\mid \xi_{k}\right),\left(\xi_{k} \mid \xi_{k}\right)=1$, where $\xi_{k}$ is a complex number. Let $\mid \xi)=\bigotimes_{k} \mid \xi_{k}$ ). These states satisfy the completeness relation:

$$
\left.\mathbb{1}=\int \prod_{k} \frac{d \xi_{k} d \bar{\xi}_{k}}{2 \pi} \mid \xi\right)(\xi \mid
$$

where $d \xi_{k} d \bar{\xi}_{k}=2 d\left(\operatorname{Re} \xi_{k}\right) d\left(\operatorname{Im} \xi_{k}\right)$.

We first consider the case where $\varphi=E \alpha, \psi=E \beta$, where $\alpha$ and $\beta$ are one particle wave functions. Using (2.7), we obtain:

$$
\begin{aligned}
\left(T_{\sigma} \varphi, T_{\sigma} \psi\right) & =\left(T_{\sigma} E \alpha, T_{\sigma} E \beta\right)=\int \prod_{k} \frac{d \xi_{k} d \bar{\xi}_{k}}{2 \pi}(E \alpha, \xi) \\
& \times(\xi, E \beta) \exp \left\{-\frac{1}{2} \sum_{k, l} \tilde{w}_{\sigma k,-l}\left(\bar{\xi}_{k} \bar{\xi}_{l}+\xi_{k} \xi_{l}\right)\right\} .
\end{aligned}
$$

$\operatorname{Now}(E \alpha, \xi)=\exp \left(\sum_{k} \bar{\alpha}_{k} \xi_{k}\right)$. 
Therefore:

$$
\begin{aligned}
\left(T_{\sigma} \varphi, T_{\sigma} \psi\right)= & \int \prod_{k} \frac{d \xi_{k} d \bar{\xi}_{k}}{2 \pi} \exp \left\{-\frac{1}{2} \sum_{k, l} \tilde{w}_{\sigma k,-l}\left(\bar{\xi}_{k} \bar{\xi}_{l}+\xi_{k} \xi_{l}\right)\right. \\
& \left.+\sum_{k} \bar{\alpha}_{k} \xi_{k}+\sum_{k} \bar{\xi}_{k} \beta_{k}\right\} .
\end{aligned}
$$

An elementary calculation of gaussian integrals gives:

$$
\begin{aligned}
\left(T_{\sigma} \varphi, T_{\sigma} \psi\right) & =\operatorname{det}\left(1-\tilde{w}_{\sigma}^{2}\right)^{-1 / 2} \exp \left\{\left(\alpha,\left(1-\tilde{w}_{\sigma}^{2}\right)^{-1} \beta\right)\right. \\
& \left.-\frac{1}{2}\left(\alpha, \tilde{w}_{\sigma}\left(1-\tilde{w}_{\sigma}^{2}\right)^{-1} \alpha^{*}\right)-\frac{1}{2}\left(\beta^{*}, \tilde{w}_{\sigma}\left(1-\tilde{w}_{\sigma}^{2}\right)^{-1} \beta\right)\right\}
\end{aligned}
$$

where $\alpha_{k}^{*}=\bar{\alpha}_{-k}$.

For $\alpha=\beta=0$, we obtain (2.5). For general $\alpha$ and $\beta$, we obtain (2.6) in the special case where $\varphi=E \alpha, \psi=E \beta$. Since the $E \alpha$ form a total set in Fock space, the result holds for general $\varphi$ and $\psi$.

The convergence problems are elementary.

When $\sigma$ tends to infinity, $\operatorname{Tr} \tilde{w}_{\sigma}^{2}$ may become infinite, and actually does for $s \geqq 4$. Then $\left\|T_{\sigma} \Omega\right\|$ tends to infinity and $T_{\sigma}$ kicks the vacuum out of Fock space. In any case (2.6) tends to a well defined limit when $\sigma$ tends to infinity, because of the above mentioned convergence properties of $\tilde{w}_{\sigma}$. This limit is easily seen to be a positive definite bilinear form on $\mathscr{D}$. We can therefore define a new scalar product on the range of $T$ by:

$$
\begin{aligned}
\left\langle\varphi_{r}, \psi_{r}\right\rangle= & \lim _{\sigma \rightarrow \infty} \frac{\left(T_{\sigma} \varphi, T_{\sigma} \psi\right)}{\left\|T_{\sigma} \Omega\right\|^{2}} \\
= & f d K d K^{\prime} d L d L^{\prime} \psi\left(K, K^{\prime}\right) \overline{\varphi\left(L, L^{\prime}\right)} E\left(-\tilde{w}\left(1-\tilde{w}^{2}\right)^{-1}\right)\left(K^{\prime}\right) \\
& \times E\left(-\tilde{w}\left(1-\tilde{w}^{2}\right)^{-1}\right)\left(L^{\prime}\right) E\left(\left(1-\tilde{w}^{2}\right)^{-1}\right)(K \mid L)
\end{aligned}
$$

where we have used the notations $\varphi_{r}, \psi_{r}$ for $T \varphi, T \psi$.

If $\left\|T_{\infty} \Omega\right\|$ is finite, then $T_{\infty}$ maps $\mathscr{D}$ into Fock space, and the new scalar product is equal to the old one, up to a multiplicative factor. If $\left\|T_{\infty} \Omega\right\|$ is infinite, then $T_{\infty}$ does not exist as a mapping from $\mathscr{D}$ to $\mathfrak{H}$, and what we are really doing is to define a new scalar product on $\mathscr{D}$.

The completion of the range of $T$ under the scalar product (2.8) gives a new Hilbert space, denoted by $\mathfrak{G}_{r}$, which is appropriate for the description of the renormalized quantities. We now proceed to define the renormalized Hamiltonian $H_{r}$, using equation (2.2). With the previous choice of $W_{\sigma}$, the RHS of (2.2) will be well behaved, whereas the vacuum self energy $-C_{\sigma}$ diverges for $s \geqq 3$ when $\sigma$ becomes infinite. We therefore subtract it from $H_{\sigma}$ before taking the limit. The matrix elements of $H_{r}$ 
are defined, for $\varphi$ and $\psi$ in $\mathscr{D}$, by:

$$
\begin{aligned}
\left\langle\varphi_{r}, H_{r} \psi_{r}\right\rangle & =\lim _{\sigma \rightarrow \infty} \frac{\left(T_{\sigma} \varphi,\left(H_{\sigma}+C_{\sigma}\right) T_{\sigma} \psi\right)}{\left\|T_{\sigma} \Omega\right\|^{2}} \\
& =\lim _{\sigma \rightarrow \infty} \frac{\left(T_{\sigma} \varphi, T_{\sigma}\left(H_{0}+V_{0 \sigma}+V_{1 \sigma}+V_{0 \sigma}-W_{\sigma}\right) \psi\right)}{\left\|\mathrm{T}_{\sigma} \Omega\right\|^{2}}
\end{aligned}
$$

The choice of $W_{\sigma}$ has eliminated the dangerous pair creation terms. The existence of the limit then follows easily from the explicit expression (2.8) for the scalar product and the convergence property of $\tilde{w}_{\sigma}$. Similarly, the following quantity tends to a finite limit when $\sigma$ tends to infinity, and is therefore bounded uniformly in $\sigma$ :

$$
\left\|\left(H_{\sigma}+C_{\sigma}\right) T_{\sigma} \varphi\right\| /\left\|T_{\sigma} \Omega\right\| .
$$

It then follows from the Riesz representation theorem that $H_{r}$ is an operator, defined on the range of $T$. This operator is clearly symmetric. We call it the renormalized Hamiltonian.

The same procedure can be applied to a large class of sufficiently regular operators, and in particular to linear functions of creation and annihilation operators.

We conclude this section with the following remark: there is some arbitrariness in the choice of $\tilde{w}_{\sigma}$ and therefore of $T_{\sigma}$. To make $H_{r}$ well defined, the condition $R=0$ is not necessary. It would be sufficient to require $R_{\infty}^{+}-W_{\infty}<\infty$ in addition to the important condition $\|\tilde{w}\|<1$. In particular, one could choose $H_{0} \perp W=V_{2}$ for $s \leqq 4$, at least for $\lambda$ sufficiently small.

\section{Renormalization Using a Bogoliubov Transformation}

The method of Section 2 did not yield any information on the selfadjointness and spectrum of $H_{r}$. Moreover, it relies heavily on the existence and properties of a $\tilde{w}$ satisfying (2.4), which is not very convenient to analyze directly. In order to circumvent these difficulties, we try to diagonalize the Hamiltonian by the use of a Bogoliubov-type transformation.

Let us define:

$$
\left\{\begin{array}{l}
b_{\sigma}(k)=a(k)+\int d l \tilde{u}_{\sigma}(k, l)\left(a(l)+a^{+}(-l)\right) \\
b_{\sigma}^{+}(k)=a^{+}(k)+\int d \dot{d} l \tilde{u}_{\sigma}(k, l)\left(a^{+}(l)+a(-l)\right)
\end{array}\right.
$$

where $\tilde{u}_{\sigma}(k, l)$ is a real symmetric kernel, possibly depending on $\sigma$ and $f$. The $b_{\sigma}^{+}$and $b_{\sigma}$ satisfy the following commutation relations:

$$
\left\{\begin{array}{l}
{\left[b_{\sigma}(k), b_{\sigma}(l)\right]=0} \\
{\left[b_{\sigma}(k), b_{\sigma}^{+}(l)\right]=\delta(k-l)+2 \tilde{u}_{\sigma}(k, l) .}
\end{array}\right.
$$


Note that (3.1) is not a Bogoliubov transformation in the usual sense, since it does not preserve the CCR. It is introduced as an intermediate step, for convenience reasons.

An elementary calculation shows that $H_{\sigma}$ can be written in the form:

where:

$$
H_{\sigma}=\int d k \omega(k) b_{\sigma}^{+}(k) b_{\sigma}(k)-D_{\sigma}
$$

$$
D_{\sigma}=\int d k d l \omega(k) \tilde{u}_{\sigma}(k, l)^{2},
$$

provided $\tilde{u}_{\sigma}$ satisfies the following equation:

$$
\frac{1}{2}(\omega(k)+\omega(l)) \tilde{u}_{\sigma}(k, l)+\int d p \omega(p) \tilde{u}_{\sigma}(k, p) \tilde{u}_{\sigma}(p, l)=\lambda \tilde{v}_{\sigma}(k, l)
$$

where:

$$
\tilde{v}_{\sigma}(k, l)=\chi_{\sigma}(k) \chi_{\sigma}(l) f(k-l)[\omega(k) \omega(l)]^{-1 / 2} .
$$

Eq. (3.3) seems to be a natural starting point for the renormalization of $H_{\sigma}$ by the method of the preceding section. We therefore introduce $T_{\sigma}$ by Eq. (2.1) and commute it successively with $b_{\sigma}$ and $b_{\sigma}^{+}$. The first step yields the identity:

$$
\begin{aligned}
b_{\sigma}(k) T_{\sigma}= & T_{\sigma}\left[a(k)+\int d l \tilde{u}_{\sigma}(k, l)\left(a(l)+a^{+}(-l)\right)\right. \\
& \left.-\int d l \tilde{w}_{\sigma}(k, l) a^{+}(-l)-\int d l d p \tilde{u}_{\sigma}(k, p) \tilde{w}_{\sigma}(p, l) a^{+}(-l)\right] .
\end{aligned}
$$

Previously, the crucial requirement was the absence of the pair creation term in the square bracket in the RHS of Eq. (2.2). We now enforce this condition by imposing the absence of the creation operator in the square bracket in the RHS of (3.7). This leads to the equation:

$$
\tilde{u}_{\sigma}(k, l)=\tilde{w}_{\sigma}(k, l)+\int d p \tilde{u}_{\sigma}(k, p) \tilde{w}_{\sigma}(p, l) .
$$

Eq. (3.5) and (3.8) will determine $\tilde{u}_{\sigma}$ and $\tilde{w}_{\sigma}$. Before actually solving them, we first prove that the kernel $\tilde{w}_{\sigma}$ thereby obtained is also a solution of the equation $R=0(2.4)$, which can be written more precisely as:

$$
\begin{aligned}
\frac{1}{2}(\omega(k)+\omega(l)) \tilde{w}_{\sigma}(k, l)=\lambda\left\{\tilde{v}_{\sigma}(k, l)-\int d p\left[\tilde{v}_{\sigma}(k, p) \tilde{w}_{\sigma}(p, l)\right.\right. \\
\left.\left.+\tilde{w}_{\sigma}(k, p) \tilde{v}_{\sigma}(p, l)\right]+\int d p d q \tilde{w}_{\sigma}(k, p) \tilde{v}_{\sigma}(p, q) \tilde{w}_{\sigma}(q, l)\right\}
\end{aligned}
$$

where $\tilde{v}_{\sigma}(k, l)$ is defined by (3.6).

In order to compare the equations, we simplify the notations. We denote by $\tilde{u}_{\sigma}, \tilde{v}_{\sigma}$ and $\tilde{w}_{\sigma}$ the operators in the one particle space $\mathfrak{G}_{1}$ defined by the corresponding kernels and by $\tilde{h}_{0}$ the restriction of $H_{0}$ to $\mathfrak{H}_{1}$. The quantities corresponding to $\sigma$ infinite will also be used later on, and will be denoted without a subscript. Eqs. (3.5), (3.8) and (3.9) can be 
rewritten respectively as:

$$
\begin{gathered}
\tilde{u}_{\sigma} \tilde{h}_{0} \tilde{u}_{\sigma}+\frac{1}{2}\left(\tilde{u}_{\sigma} \tilde{h}_{0}+\tilde{h}_{0} \tilde{u}_{\sigma}\right)=\lambda \tilde{v}_{\sigma}, \\
\tilde{u}_{\sigma}=\tilde{w}_{\sigma}+\tilde{u}_{\sigma} \tilde{w}_{\sigma}, \\
\frac{1}{2}\left(\tilde{h}_{0} \tilde{w}_{\sigma}+\tilde{w}_{\sigma} \tilde{h}_{0}\right)=\lambda\left(1-\tilde{w}_{\sigma}\right) \tilde{v}_{\sigma}\left(1-\tilde{w}_{\sigma}\right) .
\end{gathered}
$$

Multiplying (3.10) from the left and from the right by $\left(1-\tilde{w}_{\sigma}\right)$ and using (3.11), we obtain immediately (3.12). Therefore $\tilde{w}_{\sigma}$ defined through (3.10) and (3.11) solves (3.12). We next show the equality of the constants $C_{\sigma}$ (cf. (2.3)) and $D_{\sigma}$ (cf. (3.4)). They can be rewritten as:

$$
\begin{gathered}
C_{\sigma}=\lambda \operatorname{Tr} \tilde{v}_{\sigma} \tilde{w}_{\sigma}, \\
D_{\sigma}=\operatorname{Tr} \tilde{h}_{0} \tilde{u}_{\sigma}^{2} .
\end{gathered}
$$

The equality is obtained immediately by multiplying (3.10) by $\tilde{w}_{\sigma}$, taking the trace, and using (3.11) and the commutativity of $\tilde{u}_{\sigma}$ and $\tilde{w}_{\sigma}$. The preceding arguments will become rigorous after we have solved (3.10). These two results exhibit the connection between the two renormalization procedures.

We now turn to the analysis of (3.10). It can be rewritten as:

where:

$$
\tilde{u}_{\sigma}^{\prime 2}+\tilde{u}_{\sigma}^{\prime} \tilde{g}^{1 / 2}+\tilde{g}^{1 / 2} \tilde{u}_{\sigma}=\lambda \tilde{f_{\sigma}}
$$

$$
\left\{\begin{aligned}
\tilde{u}_{\sigma}^{\prime} & =\tilde{h}_{0}^{1 / 2} \tilde{u}_{\sigma} \tilde{h}_{0}^{1 / 2}, \\
\tilde{g} & =\frac{1}{4} \tilde{h}_{0}^{2}, \\
\tilde{f}_{\sigma} & =\tilde{h}_{0}^{1 / 2} \tilde{v}_{\sigma} \tilde{h}_{0}^{1 / 2} .
\end{aligned}\right.
$$

More explicitly:

$$
\tilde{f_{\sigma}}(k, l)=\chi_{\sigma}(k) f(k-l) \chi_{\sigma}(l)
$$

(3.15) is solved by:

$$
\tilde{u}_{\sigma}^{\prime}=\left(\tilde{g}+\lambda \tilde{f_{\sigma}}\right)^{1 / 2}-\tilde{g}^{1 / 2} .
$$

The operator $\tilde{g}$ is self adjoint and bounded from below by $\frac{1}{4} \mathrm{~m}^{2} ; \tilde{f}_{\sigma}$ is a bounded positive operator, with $\left\|\tilde{f}_{\sigma}\right\| \leqq 1$. Therefore, for any real $\lambda$, $\tilde{g}+\lambda \tilde{f}_{\sigma}$ is self adjoint on the domain of $\tilde{g}$, and bounded from below by $\min \left(\frac{1}{4} m^{2}, \frac{1}{4} m^{2}+\lambda\right)$. For $\lambda>-\frac{1}{4} m^{2}, \tilde{g}+\lambda \tilde{f}_{\sigma}$ is strictly positive, and its square root is a well defined self adjoint (positive) operator. We shall prove in appendix 2, that $\tilde{u}_{\sigma}^{\prime}$ and $\tilde{u}_{\sigma}$ defined by (3.17) and (3.16) have the following properties: $\tilde{u}_{\sigma}$ and $\tilde{u}_{\sigma}^{\prime}$ are bounded operators in $\mathfrak{H}_{1}$, and $\tilde{u}_{\sigma}>-1 / 2$. All the bounds are uniform in $\sigma \leqq \infty$. These operators are represented by real symmetric $\mathscr{C}^{\infty}$ bounded kernels. For finite $\sigma$, the 


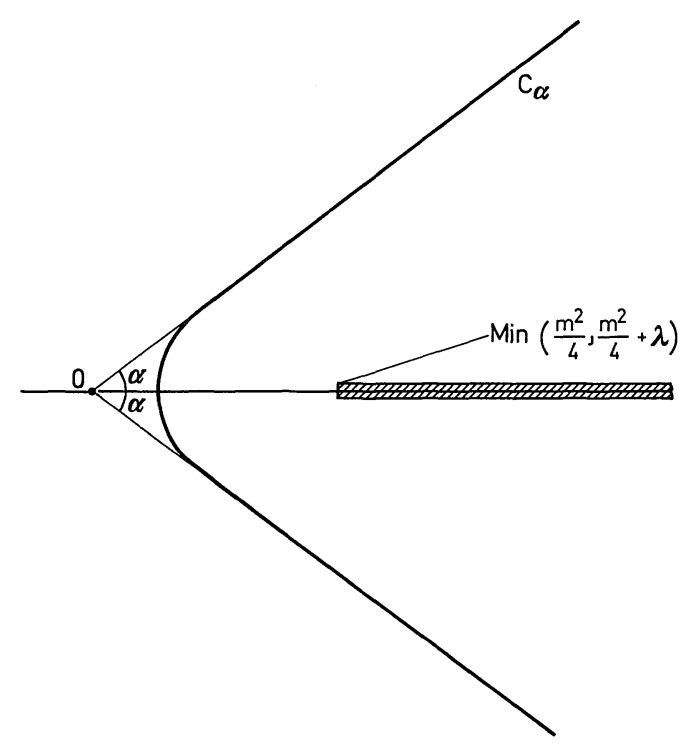

Fig. 1. The integration contour in (3.18) and (A2.1, 2, 3)

kernels have compact support; for $\sigma$ infinite, the kernels are rapidly decreasing functions of each variable when the other is held fixed. When $\sigma$ tends to infinity, $\tilde{u}_{\sigma}$ and $\tilde{u}_{\sigma}^{\prime}$ tend to $\tilde{u}$ and $\tilde{u}^{\prime}$ in the strong operator topology. All these properties are easily obtained from a perturbation expansion of (3.15) for $|\lambda|<\frac{1}{4} m^{2}$, and will be derived in Appendix 2 for any real $\lambda>-\frac{1}{4} m^{2}$ by means of the integral representation:

$$
\tilde{u}_{\sigma}^{\prime}=\frac{\lambda}{2 \pi i} \int_{C_{\alpha}} z^{1 / 2} d z\left(z-\tilde{g}-\lambda \tilde{f}_{\sigma}\right)^{-1} \tilde{f}_{\sigma}(z-\tilde{g})^{-1}
$$

where $C_{\alpha}$ is the contour shown in Fig. 1 .

We can now define $\tilde{w}_{\sigma}$ by:

$$
\tilde{w}_{\sigma}=\tilde{u}_{\sigma}\left(1+\tilde{u}_{\sigma}\right)^{-1} \equiv\left(1+\tilde{u}_{\sigma}\right)^{-1} \tilde{u}_{\sigma},
$$

$\tilde{w}_{\sigma}$ has all the properties stated in Section 2; in particular $\left\|\tilde{w}_{\sigma}\right\|<1$ follows from $\tilde{u}_{\sigma}>-1 / 2$ uniformly in $\sigma$ (see Appendix 2). These results complete the arguments given in Section 2.

We now come back to the Bogoliubov transformation. The previous relation (3.1) between the $\left(a, a^{+}\right)$and the $\left(b_{\sigma}, b_{\sigma}^{+}\right)$did not preserve the $\mathrm{CCR}$. These are easily recovered through a second transformation defined by:

$$
c_{\sigma}(k)=\int d l\left(1+2 \tilde{u}_{\sigma}\right)^{-1 / 2}(k, l) b_{\sigma}(l) .
$$


The operator $\left(1+2 \tilde{u}_{\sigma}\right)^{-1 / 2}$ is bounded uniformly in $\sigma$ and converges strongly to $(1+2 \tilde{u})^{-1 / 2}$ for $\sigma \rightarrow \infty$.

The hamiltonian can be expressed in terms of the $\left(c_{\sigma}, c_{\sigma}^{+}\right)$as:

$$
H_{\sigma}+C_{\sigma}=\int d k d l \tilde{h}_{\sigma}(k, l) c_{\sigma}^{+}(k) c_{\sigma}(l)
$$

where:

$$
\tilde{h}_{\sigma}=\left(1+2 \tilde{u}_{\sigma}\right)^{1 / 2} \tilde{h}_{0}\left(1+2 \tilde{u}_{\sigma}\right)^{1 / 2} .
$$

These expressions suggest a new definition of a renormalized Hamiltonian. Let $\left(a^{\prime}(k), a^{\prime+}(k)\right)$ be a representation of the CCR in the Fock space $\mathfrak{S}^{\prime}$. We define a renormalized Hamiltonian for the theory by:

$$
H^{\prime}=\int d k d l \tilde{h}(k, l) a^{+}(k) a^{\prime}(l)
$$

where:

$$
\tilde{h}=(1+2 \tilde{u})^{1 / 2} \tilde{h}_{0}(1+2 \tilde{u})^{1 / 2} .
$$

$H^{\prime}$ is a self adjoint positive operator, and has the no particle state $\Omega^{\prime}$ as a unique ground state.

We now compare the two renormalization procedures. For this purpose, we define a transformation $S$ from $\mathscr{D} \subset \mathfrak{S}$ to $\mathfrak{H}^{\prime}$ by:

$$
\begin{aligned}
S \varphi(K) \equiv & \varphi^{\prime}(K)=f d K^{\prime} d L E\left(\left(1-\tilde{w}^{2}\right)^{-1 / 2}\right)\left(K \mid K^{\prime}\right), \\
& E\left(-\tilde{w}\left(1-\tilde{w}^{2}\right)^{-1}\right)(L) \varphi\left(K^{\prime}, L\right) .
\end{aligned}
$$

The range of $S$ is immediately seen to be dense in $\mathfrak{S}^{\prime}$. Furthermore:

$$
\left\langle\varphi_{r}, \psi_{r}\right\rangle=(S \varphi, S \psi) \equiv\left(\varphi^{\prime}, \psi^{\prime}\right)
$$

for all $\varphi, \psi \in \mathscr{D}$. Therefore, we can identify $\mathfrak{H}_{r}$ and $\mathfrak{H}^{\prime}$, by identifying the images $\varphi_{r}$ and $\varphi^{\prime}$ of the same $\varphi$ for all $\varphi$ belonging to $\mathscr{D}$.

In the same way as we have defined $H_{r}$ in the space $\mathfrak{G}_{r}$, we also define the operators $c_{r}$ in $\mathfrak{G}_{r}$ by:

$$
\left\langle\varphi_{r}, c_{r}(k) \psi_{r}\right\rangle=\lim _{\sigma \rightarrow \infty} \frac{\left(T_{\sigma} \varphi, c_{\sigma}(k) T_{\sigma} \psi\right)}{\left\|T_{\sigma} \Omega\right\|^{2}} .
$$

The adjoint $c_{r}^{+}(k)$ is defined similarly.

We now prove that the identification of $\mathfrak{H}_{r}$ with $\mathfrak{H}^{\prime}$, identifies $c_{r}$ with $a^{\prime}$. In fact:

$$
\begin{aligned}
\left\langle\varphi_{r}, c_{r}(k) \psi_{r}\right\rangle & =\lim _{\sigma \rightarrow \infty} \frac{\left(T_{\sigma} \varphi, T_{\sigma} \int d l\left[\left(1+2 \tilde{u}_{\sigma}\right)^{-1 / 2}\left(1+\tilde{u}_{\sigma}\right)\right](k, l) a(l) \psi\right)}{\left\|T_{\sigma} \Omega\right\|^{2}} \\
& =\lim _{\sigma \rightarrow \infty} \frac{\left(T_{\sigma} \varphi, T_{\sigma} \int d l\left(1-\tilde{w}_{\sigma}^{2}\right)^{-1 / 2}(k, l) a(l) \psi\right)}{\left\|T_{\sigma} \Omega\right\|^{2}} \\
& =\left(S \varphi, S \int d l\left(1-\tilde{w}^{2}\right)^{-1 / 2}(k, l) a(l) \psi\right)
\end{aligned}
$$


by (3.26). Now:

$$
\begin{aligned}
\left(S \int\right. & \left.d l\left(1-\tilde{w}^{2}\right)^{-1 / 2}(k, l) a(l) \psi\right)(K) \\
= & f d K^{\prime} d L d l\left(1-\tilde{w}^{2}\right)^{-1 / 2}(k, l) E\left(\left(1-\tilde{w}^{2}\right)^{-1 / 2}\right)\left(K \mid K^{\prime}\right) \\
& E\left(-\tilde{w}\left(1-\tilde{w}^{2}\right)^{-1 / 2}\right)(L) \psi\left(K^{\prime}, L, l\right) \\
= & f d K^{\prime \prime} d L E\left(\left(1-\tilde{w}^{2}\right)^{-1 / 2}\right)\left(K, k \mid K^{\prime \prime}\right) E\left(-\tilde{w}\left(1-\tilde{w}^{2}\right)^{-1}\right)(L) \psi\left(K^{\prime \prime}, L\right) \\
= & \left(a^{\prime}(k) S \psi\right)(K) .
\end{aligned}
$$

From the explicit expression of the scalar product, the regularity and convergence properties of $\tilde{u}_{\sigma}$, it follows by the same type of computation that for any $\varphi, \psi \in \mathscr{D}$ :

$$
\left\langle\varphi_{r}, H_{r} \psi_{r}\right\rangle=\left(\varphi^{\prime}, H^{\prime} \psi^{\prime}\right)
$$

This relation completes the identification of the two renormalization procedures.

\section{Removal of the Space Cut-off}

Until now, the space cut-off $f$ has been kept fixed, and we have removed the UV cut-off $\sigma$. The ensuing situation is the following:

- The Hilbert space $\mathfrak{G}_{r}$ is the Fock space of the CCR for the operators $c_{r}(k)$ and $c_{r}^{+}(k)$.

- The Hamiltonian $H_{r}$ is the positive operator:

$$
H_{r}=\int d k d l \tilde{h}(k, l) c_{r}^{+}(k) c_{r}(l)
$$

where $\tilde{h}$ is defined by (3.24), and its (unique) ground state is the no particle state $\Omega_{r}$.

- The renormalized field at an arbitrary time $t$ is given by:

$$
\begin{aligned}
\Phi_{r}(x, t)= & (2 \pi)^{-s / 2} \int d k d l[2 \omega(k)]^{-1 / 2} e^{i k x} \\
& \times\left\{\left((1+2 \tilde{u})^{-1 / 2} e^{-i t \tilde{h}}\right)(k, l) c_{r}(l)\right. \\
& +\left((1+2 \tilde{u})^{-1 / 2} e^{i t \tilde{h}}\right)(k, l) c_{r}^{+}(-l\} .
\end{aligned}
$$

This formula is obtained as follows: for finite $\sigma$, the time evolution of the $c_{\sigma}(k)$ is given by:

$$
c_{\sigma}(k, t)=e^{i t H_{\sigma}} c_{\sigma}(k) e^{-i t H_{\sigma}}
$$

or equivalently by:

$$
c_{\sigma}(k, t)=\int d l e^{-i t \tilde{h}_{\sigma}}(k, l) c_{\sigma}(l)
$$

where $\tilde{h}_{\sigma}$ is defined by (3.22). 
The $a(k)$ and $a^{+}(k)$ are expressed as functions of $c_{\sigma}$ and $c_{\sigma}^{+}$by:

$$
\begin{aligned}
a(k)= & \int d l\left\{\left[\left(1+2 \tilde{u}_{\sigma}\right)^{-1 / 2}\left(1+\tilde{u}_{\sigma}\right)\right](k, l) c_{\sigma}(l)\right. \\
& \left.-\left[\left(1+2 \tilde{u}_{\sigma}\right)^{-1 / 2} \tilde{u}_{\sigma}\right](k, l) c_{\sigma}^{+}(-l)\right\} \\
a^{+}(k)= & \int d l\left\{\left[\left(1+2 \tilde{u}_{\sigma}\right)^{-1 / 2}\left(1+\tilde{u}_{\sigma}\right)\right](k, l) c_{\sigma}^{+}(l)\right. \\
& \left.-\left[\left(1+2 \tilde{u}_{\sigma}\right)^{-1 / 2} \tilde{u}_{\sigma}\right](k, l) c_{\sigma}(-l)\right\} .
\end{aligned}
$$

Substituting $(4.5,6)$ and $(4.4)$ in the expressions of the field in terms of $a$ and $a^{+}$, and taking the limit of infinite $\sigma$ as in section 2, we obtain (4.2). The only non trivial point is to prove the strong convergence of:

$$
\left(1+2 \tilde{u}_{\sigma}\right)^{-1 / 2} e^{ \pm i t \tilde{h}_{\sigma}}
$$

in $\mathfrak{H}_{1}$ when $\sigma$ tends to infinity. This is done in appendix 2 .

We now turn to the elimination of the space-cut off $f$. The Hilbert space $\mathfrak{S}_{r}$ and the vacuum state $\Omega_{r}$ do not depend on $f$, whereas the field operator $\Phi_{r}(x, t)$ and the Hamiltonian $H_{r}$ contain $f$ through the function $\tilde{u}$. We now show that, when $f$ tends to $\delta$ in the Schwartz space $\mathscr{S}^{\prime}$, the smeared field converges strongly on any reasonable domain to the free field with mass $\left(m^{2}+4 \lambda\right)^{1 / 2}$. This conclusion follows immediately once we know that:

$$
(1+2 \tilde{u})^{-1 / 2} e^{ \pm i t \tilde{h}}
$$

converges strongly in $\mathfrak{S}_{1}$ to the operator that consists in the multiplication by:

$$
\left(\frac{k^{2}+m^{2}}{k^{2}+m^{2}+4 \lambda}\right)^{1 / 4} e^{ \pm i t\left(k^{2}+m^{2}+4 \lambda\right)^{1 / 2}}
$$

This is proved in Appendix 2.

As a by-product, we also obtain the strong convergence of the unitary group $\exp \left(i t H_{r}\right)$.

We finally end up, as expected, with a free field theory, with the renormalized mass.

\section{Appendix 1. Self Adjointness of the Cut-off Hamiltonian}

Let us consider the Hamiltonian $H=H_{0}+V$ where $H_{0}$ and $V$ are defined respectively by (2.0) and by:

$$
V=\int d k d l r(k, l) a^{+}(k) a(l)+\int d k d l s(k, l)\left(a(k) a(l)+a^{+}(k) a^{+}(l)\right) .
$$

The functions $r$ and $s$ are square integrable and real symmetric. It is clear that $H$ is a symmetric operator on the domain of $H_{0}$. 
We want to prove that $H$ is essentially self adjoint on the domain $\mathscr{D}$ defined in section 2. For this purpose, it is sufficient to check that $(z-H) \mathscr{D}$ is dense in Fock space for any non real $z$. This amounts to show that, if for all $\varphi$ in $\mathscr{D}$,

then $\psi=0$.

$$
(\psi,(z-H) \varphi)=0
$$

Let $\psi$ satisfy (A 1.2) for all $\varphi \in \mathscr{D}$. If $\varphi$ belongs to the $n$ particle subspace $\mathfrak{H}_{n}$, (A 1.2) implies that $\psi_{n}$ is in the domain of $H_{0}$, because the restriction of $V$ to $\mathfrak{S}_{n}$ is a bounded operator. Here, $\psi_{n}$ is the projection of $\psi$ on $\mathfrak{H}_{n}$. Then (A 1.2) implies that the projection on every $\mathfrak{H}_{n}$ of the vector $(z-H) \psi$ is zero. In particular, for all $n$, we have:

$$
\left(\psi_{n},\left(H_{0}+V-z\right) \psi\right)=0 .
$$

Taking the imaginary part, we obtain:

$$
\operatorname{Im} z\left\|\psi_{n}\right\|^{2}=\operatorname{Im}\left[\left(\psi_{n}, V_{0} \psi_{n+2}\right)+\left(\psi_{n}, V_{2} \psi_{n-2}\right)\right]
$$

where $V_{0}$ and $V_{2}$ are the pair annihilation and the pair creation terms of $V$. We sum over $n$ :

$$
\operatorname{Im} z \sum_{n=0}^{N}\left\|\psi_{n}\right\|^{2}=\operatorname{Im}\left[\left(\psi_{N}, V_{0} \psi_{N+2}\right)+\left(\psi_{N-1}, V_{0} \psi_{N+1}\right)\right] .
$$

Applying Schwartz' inequality, we get

$$
\begin{aligned}
|\operatorname{Im} z|(N+2)^{-1} \sum_{n=0}^{N}\left\|\psi_{n}\right\|^{2} \leqq \frac{1}{2}\|s\|_{2}\left\{\left\|\psi_{N}\right\|^{2}+\left\|\psi_{N+2}\right\|^{2}\right. \\
\left.+\left\|\psi_{N-1}\right\|^{2}+\left\|\psi_{N+1}\right\|^{2}\right\} .
\end{aligned}
$$

This inequality shows that for $\operatorname{Im} z \neq 0,\|\psi\|^{2} \equiv \sum_{N=0}^{\infty}\left\|\psi_{N}\right\|^{2}$ cannot be finite unless $\psi=0$.

\section{Appendix 2. Properties of $\tilde{u}_{\sigma}$ and Related Quantities}

In this appendix, we analyze the solution of (3.15) and related operators needed throughout the paper. For this purpose, we shall use the following integral representation, which holds for any strictly positive operator $A$ and for all $\psi$ in the domain of $A$ :

$$
A^{1 / 2} \psi=\frac{1}{2 \pi i} \int_{C_{\alpha}} d z z^{1 / 2}(z-A)^{-1} z^{-1} A \psi
$$

where $C_{\alpha}$ is the contour shown on Fig. 1 . 
The integral converges absolutely, is independent of the contour in any reasonable sense and is easily seen, by the use of the spectral decomposition of $A$, to represent the left-hand side. Applying (A2.1) to the operators $\tilde{g}+\lambda \tilde{f_{\sigma}}$ and $\tilde{g}$, we can rewrite (3.17) for real $\lambda>-\frac{1}{4} m^{2}$, as:

$$
\tilde{u}_{\sigma}^{\prime}=\frac{1}{2 \pi i} \int_{C_{\alpha}} d z z^{-1 / 2}\left[\left(z-\tilde{g}-\lambda \tilde{f_{\sigma}}\right)^{-1}\left(\tilde{g}+\lambda \tilde{f_{\sigma}}\right)-(z-\tilde{g})^{-1} \tilde{g}\right]
$$

where both sides are defined on the domain of $\tilde{g}$, or equivalently:

$$
\tilde{u}_{\sigma}^{\prime}=\frac{\lambda}{2 \pi i} \int_{\mathcal{C}_{\alpha}} d z z^{1 / 2}\left(z-\tilde{g}-\lambda \tilde{f_{\sigma}}\right)^{-1} \tilde{f_{\sigma}}(z-\tilde{g})^{-1} .
$$

It is easily seen that this operator is bounded, with a bound independent of both cut offs. Moreover, it is a continuous function of $\tilde{f}_{\sigma}$ in the strong operator topology; in fact, the integrand is uniformly bounded in norm by an integrable function, and is a strongly continuous function of $\tilde{f}_{\sigma}$ since each factor is strongly continuous and uniformly bounded. The strong continuity of $\tilde{u}_{\sigma}^{\prime}$ then follows from Lebesgue's theorem. Because of (3.16), the same properties hold for $\tilde{u}_{\sigma}$.

We next prove that $\tilde{u}_{\sigma}+\frac{1}{2}$ is strictly positive uniformly in $\sigma$ and $f$. In fact, let $\tilde{x}=2\left(\tilde{g}+\lambda \tilde{f_{\sigma}}\right)^{1 / 2}$. Then

$$
\tilde{x}^{2} \equiv 4 \tilde{g}+4 \lambda \tilde{f}_{\sigma} \geqq \operatorname{Min}\left(1,1+\frac{4 \lambda}{m^{2}}\right) \tilde{h}_{0}^{2} .
$$

Therefore $\tilde{x}$ has a bounded inverse, and

where:

$$
\left\|\tilde{h}_{0} \tilde{x}^{-1}\right\|=\left\|\tilde{x}^{-1} \tilde{h}_{0}\right\| \leqq M(\lambda)
$$

Now:

$$
M(\lambda)=\operatorname{Max}\left(1,\left(1+\frac{4 \lambda}{m^{2}}\right)^{-1 / 2}\right)
$$

$$
\tilde{h}_{0}^{1 / 2} \tilde{x}^{-1} \tilde{h}_{0}^{1 / 2} \equiv \tilde{h}_{0} \tilde{x}^{-1}\left(1+\left[\tilde{x}-\tilde{h}_{0}, \tilde{h}_{0}^{-1 / 2}\right] \tilde{x}^{-1} \tilde{h}_{0}^{1 / 2}\right)
$$

On the other hand:

Consequently:

$$
\tilde{u}_{\sigma}+\frac{1}{2}=\frac{1}{2}\left(\tilde{h}_{0}^{-1 / 2} \tilde{x} \tilde{h}_{0}^{-1 / 2}\right)
$$

$$
\left\|\left(\tilde{u}_{\sigma}+\frac{1}{2}\right)^{-1}\right\| \leqq 2 M(\lambda)\left(1+4 M(\lambda) m^{-1}\left\|\tilde{u}_{\sigma}^{\prime}\right\|\right) .
$$

It is important to remark that this bound can be made cut off independent, since $\left\|\tilde{u}_{\sigma}^{\prime}\right\|$ is bounded uniformly with respect to both cut offs. 
We now turn to prove that $\tilde{u}_{\sigma}^{\prime}$ can be represented by a regular kernel. In fact, after extraction of the lowest order term $2 \lambda \tilde{f}_{\sigma}(k, l)[\omega(k)+\omega(l)]^{-1}$, we are left with the following remainder:

$$
\frac{\lambda^{2}}{2 \pi i} \int_{C_{\alpha}} d z z^{-1 / 2}(z-\tilde{g})^{-1} \tilde{f_{\sigma}}\left(z-\tilde{g}-\lambda \tilde{f_{\sigma}}\right) \tilde{f_{\sigma}}(z-\tilde{g})^{-1} .
$$

In order to derive its properties, it will be sufficient to prove that the operator $\left(z-\tilde{g}-\lambda \tilde{f_{\sigma}}\right)^{-1}$ maps $\mathscr{S}$ into $\mathscr{S}$. Let $\psi \in \mathscr{S}$ and

$$
\varphi=\left(z-\tilde{g}-\lambda \tilde{f}_{\sigma}\right)^{-1} \psi
$$

or:

$$
\psi(k)=\left(z-\frac{\omega(k)^{2}}{4}\right) \varphi(k)-\lambda \int d l \tilde{f}_{\sigma}(k, l) \varphi(l) .
$$

The function $\varphi$ is obviously $\mathscr{C}^{\infty}$ because of the explicit form of $\tilde{f_{\sigma}}$. The following remarks show that $\varphi$ is rapidly decreasing. The last term in the RHS of (A 2.5) is uniformly bounded in $k$, because of Schwartz inequality. Therefore $\omega(k)^{2} \varphi(k)$ is bounded. Multiplying (A2.5) by $\omega(k)^{2}$ and using the inequality:

$$
\omega(k) \leqq C t \omega(l) \omega(k-l)
$$

we prove that $\omega(k)^{4} \varphi$ is also bounded. This process can be iterated and applied to all derivatives of $\varphi$. Consequently $\varphi \in \mathscr{S}$. This result implies that when $\sigma$ is finite, the kernel of $\tilde{u}_{\sigma}^{\prime}$ is $\mathscr{C}^{\infty}$ with compact support. For $\sigma$ infinite, $\tilde{f}$ reduces to the convolution with $f$, and the kernel $\tilde{u}^{\prime}$ belongs to $\mathscr{S}$ as a function of each variable when the other is held fixed.

The properties of $\tilde{w}_{\sigma}$ stated in section 2 follow easily from the corresponding properties of $\tilde{u}_{\sigma}$ and the relation (3.19). In particular, the regularity of the kernel is exhibited in the partial expansion:

$$
\tilde{w}_{\sigma}=\tilde{u}_{\sigma}-\tilde{u}_{\sigma}\left(1+\tilde{u}_{\sigma}\right)^{-1} \tilde{u}_{\sigma} .
$$

Furthermore, $\tilde{w}_{\sigma}$ is a strongly continuous function of $\tilde{u}_{\sigma}$ and therefore converges strongly as $\sigma$ tends to infinity.

Similarly, the operators $\left(1+2 \tilde{u}_{\sigma}\right)^{ \pm 1 / 2}$ converge strongly as $\sigma$ tends to infinity and/or $f$ tends to $\delta$, because the operator $1+2 \tilde{u}_{\sigma}$ is uniformly bounded away from zero.

We next prove the strong convergence of $\tilde{h}_{\sigma}$ on the domain of $\tilde{h}_{0}$ as $\sigma$ tends to infinity and/or $f$ tends to $\delta$. The operator $\tilde{h}_{\sigma}$ can be rewritten as:

$$
\begin{aligned}
& \tilde{h}_{\sigma} \equiv\left(1+2 \tilde{u}_{\sigma}\right)^{1 / 2} \tilde{h}_{0}\left(1+2 \tilde{u}_{\sigma}\right)^{1 / 2} \\
& =\left(1+2 \tilde{u}_{\sigma}\right) \tilde{h}_{0}+\left(1+2 \tilde{u}_{\sigma}\right)^{1 / 2}\left[\tilde{h}_{0},\left(1+2 \tilde{u}_{\sigma}\right)^{1 / 2}\right] .
\end{aligned}
$$


The first term in the last member of (A2.6) converges strongly on the domain of $\tilde{h}_{0}$. The commutator in the second term is seen by the use of Cauchy's formula for $\left(1+2 \tilde{u}_{\sigma}\right)^{1 / 2}$ to be a uniformly bounded strongly continuous function of $\left[\tilde{h}_{0}, \tilde{u}_{\sigma}\right]$ provided the latter is uniformly bounded. By the same method using the representation (A2.3), it is sufficient to ensure the uniform boundedness and strong convergence of $\left[\tilde{h}_{0}, \tilde{f}_{\sigma}\right]$. This last property holds in particular when $\sigma$ tends to infinity for fixed $f$ and when $f$ tends to $\delta$ in $\mathscr{S}^{\prime}$.

A theorem of Trotter [9] then implies the strong convergence of the unitary group $e^{ \pm i t \tilde{h}_{\sigma}}$ as $\sigma$ tends to infinity and/or $f$ tends to $\delta$. As a consequence, the unitary group $\exp \left(i t H_{r}\right)$ converges strongly in $\mathfrak{H}_{r}$ as $f$ tends to $\delta$.

Finally, the limiting expression for $\tilde{u}$ when $f=\delta$ is easily computed to be:

$$
\tilde{u}(k, l)=\frac{1}{2}\left[\left(\frac{k^{2}+m^{2}+4 \lambda}{k^{2}+m^{2}}\right)^{1 / 2}-1\right] \delta(k-l) .
$$

\section{References}

1. Glimm, J.: Commun. Math. Phys. 5, 343 (1967); 6, 61 (1967).

2. - Commun. Math. Phys. 10, 1 (1968).

3. Guénin, M., Velo, G.: Helv. Phys. Acta 41, 362 (1968).

4. Hepp, K.: Colloque international du CNRS, Gif sur Yvette (1969).

5. Friedrichs, K. O.: Perturbation of spectra in Hilbert space. Am. Math. Soc., Providence (1965).

6. Glimm, J.: Lecture notes in Varenna, 1968.

7. Ginibre, J.: Seminar notes on Euclidean Quantum Field Theory, IHES (1966).

8. Glauber, R. J.: Phys. Rev. 131, 2766 (1963).

9. Nelson, E.: Operator differential equations. Princeton University lecture notes, 1965.

\section{J. Ginibre}

Laboratoire de Physique Théorique

et Hautes Energies,

Bât. 211, Fac. des Sciences,

91 Orsay, France
G. Velo

Istituto di Fisica A. Righi,

Università di Bologna,

Via Irnerio 46,

Bologna 40126, Italy 